\title{
Theoretical Proposals on How Vertical Harmony May Convey Nostalgia and Longing in Music
}

\author{
IMRE LAHDELMA[1] \\ Department of Music, University of Jyväskylä, Finland \\ TUOMAS EEROLA \\ Department of Music, Durham University, UK
}

\begin{abstract}
Music is often associated with the emotions of nostalgia and longing. According to previous survey studies, both nostalgia and longing are among the most common emotions evoked by music (Juslin, 2011). Despite nostalgia's significance as a musical emotion, research on the specific properties of music that might contribute to this particular emotion has been scarce. A recent empirical experiment by Lahdelma and Eerola (2014) sought to explore whether single chords could be effective at conveying musical emotions to listeners, which spanned complex emotions such as nostalgia/longing. According to the results, single chords such as the minor triad, the minor seventh and especially the major seventh communicated the emotion of nostalgia effectively. The aim of the current paper is to raise several possible explanations that might account for the ability of single chords to convey the emotion of nostalgia. In these explanations we consider cultural, music-theoretical and psychoacoustic issues, as well as their possible interactions. The three proposed candidate explanations are (1) learning, (2) intrinsic emotional connotations arising from tonal relations, and (3) clashing conventions arising from concurrent yet separate affective associations, stemming from certain triad and interval combinations. Finally, we propose experimental designs for future research to empirically test these explanations.
\end{abstract}

Submitted 2014 November 17; accepted 2015 September 8.

KEYWORDS: chord, vertical harmony, emotion, nostalgia, longing

NOSTALGIA is a common emotion (Boym, 2001), described as a universal experience that transcends age groups and is present across the lifespan (Sedikides, Wildschut, \& Baden, 2004). According to Wildschut, Sedikides, and Routledge (2008), the term nostalgia "derives from the Greek words nostos (return) and algos (pain)" (p. 20), literally meaning "the suffering evoked by the desire to return to one's place of origin" (p. 20). The New Oxford Dictionary of English (1998) defines nostalgia as "a sentimental longing or wistful affection for the past" (p. 1266), underlining the longing quality inherent in the emotion. According to Batcho (2007), "most theorists agree that the distinctive emotional character of nostalgia is bittersweet - a mix of sadness and wistful joy" (p. 362). Sedikides, Wildschut, Arndt, and Routledge (2008) propose that "nostalgia is triggered by dysphoric states such as negative mood and loneliness" (p. 304), but that it also generates positive affect. Nostalgia's positive effects have also been reported by, for example, Juhl and Routledge (2013) and Cheung et al. (2013).

These positive effects could be one reason why music is often called for to induce nostalgia, as music is a common source of nostalgia (e.g., Barrett et al., 2010; Zentner, Grandjean, \& Scherer, 2008), and it often serves a nostalgic function in everyday life (Juslin, Harmat, \& Eerola, 2013). Juslin (2011) proposes that on the basis of previous survey studies both nostalgia and longing are among the most common emotions evoked by music, and nostalgia's prominence among music-induced feelings has also been pointed out by, for example, Zentner and Eerola (2010). In a recent study by Taruffi and Koelsch (2014), nostalgia was indicated as the most frequent emotion evoked by sad music among a large and heterogeneous pool of participants. Interestingly, the prevalence of nostalgia in music is not only limited to felt emotional experiences, since it is common also as a perceived emotion in music (Juslin \& Laukka, 2004). 
The bittersweet component of nostalgia is present in its equivalent musical emotion. Zentner et al. (2008) suggest that musical emotions have a tendency to occur in a blended manner, and that these blended or mixed emotions can have features of both positive and negative qualities - nostalgia being a good example of an emotion encompassing a variety of closely related terms, including wistfulness and longing. For this reason, it is not surprising that many scholars have labelled this emotion with a dual label such as nostalgia/longing (e.g., Juslin, Liljeström, Västfjäll, Barradas, \& Silva, 2008), and the two terms are being used more or less interchangeably in emotion questionnaires and subsequent factor structures derived from them (e.g., Zentner et al., 2008). Moreover, in music literature (e.g., Cooke, 1959; Mitchell, 2002) the terms "nostalgia" and "longing" are often also used interchangeably. To simplify the terminology, we will label this complex and blended emotion as nostalgia to encompass the most common variants, such as nostalgia/longing and wistfulness. We will return to the terminology question in the discussion section.

Despite nostalgia's significance as a musical emotion, research on the specific properties of music that contribute to this particular emotion has been scarce. Previous literature (Barrett et al., 2010; Janata, Tomic, \& Rakowski, 2007; Wildschut, Sedikides, Arndt, \& Routledge, 2006) suggests that the source of music-evoked nostalgic experience is in the idiosyncratic associations that people have formed between certain music and events in their past. Juslin and Västfjäll (2008) describe the term episodic memory as referring "to a process whereby an emotion is induced in a listener because the music evokes a memory of a particular event in the listener's life" (p. 567). While the case of idiosyncratic associations as a source of musical nostalgia may well explain the case of music-evoked, felt nostalgia, it does not take into account the possibility that music by itself could be able to convey and express nostalgia. Survey results have suggested that music is able to express nostalgia (e.g., Juslin \& Laukka, 2004), although this does not rule out that memories would be the underlying cause. However, the empirical evidence that hints at the ability of unfamiliar music to convey nostalgia is scarce. A recent result from a cross-cultural comparison implies that listeners are able to recognize longing in improvised performances across cultures (Laukka, Eerola, Thingujam, Yamasaki, \& Beller, 2013).

When we look at the musicological descriptions of pieces, music scholars often resort to the terms of nostalgia or longing. For example, Cooke (1959) mentions Frederick Delius depicting "obsessive nostalgia" (p. 76) in his music, Freeman (1992) describes Richard Wagner's music as the "language of longing", and Tagg (1993) uses the term nostalgia to depict musical moods. While some attempts have been made to depict certain musical structures conveying nostalgia (e.g., Key, 1995; Malin, 2006), this claim has remained contentious. A recent empirical experiment by Lahdelma and Eerola (2014) sought to explore whether single, isolated chords could be effective at conveying musical emotions to listeners, which spanned complex, mixed emotions such as nostalgia. Intriguingly, the results suggest that particular chords consistently convey nostalgia to listeners. In other words, the results imply that nostalgia could be considered as an inherent feature in music, and that vertical harmony might play a role in conveying this particular emotion. This is not only surprising in terms of inherent or learnt associations with music, but the brevity and the lack of musical context of the actual stimuli rule out musically complex explanations: in music, emotions are often seen as requiring time to unfold (e.g., Meyer, 1956). However, Schubert (2010) notes that "this is not mandatory to its definition, since an emotion can be detected in a static image or object such as a photograph" (p. 223). Also, studies by, for example, Bigand, Vieillard, Madurell, Marozeau, and Dacquet (2005), Eerola, Alluri, and Ferrer (2012), and Filipic, Tillmann, and Bigand (2010), suggest that extremely brief $(<1 \mathrm{~s}$, or even $<250 \mathrm{~ms}$ ) musical excerpts have the ability to communicate clear emotional signals. Even though it is widely held in music theory that single chords have musical significance only in relation to other chords (e.g., Hindemith, 1942), it is worth noting that even single chords in isolation are able to elicit brain responses that can be broadly discriminated in terms of emotions (e.g., Bakker \& Martin, 2015; Pallesen et al., 2005).

The aim of the current study is to discuss how musical structures, particularly vertical harmony, could convey the complex, mixed emotion of nostalgia, the focus being on the perception of nostalgia as opposed to the induction of this particular emotion, and the framework being exclusively Western music. In this paper, we focus on chords since this is the only musical feature that has been linked with nostalgia in an empirical setting so far. We will also demonstrate the connection with concrete musical examples, drawn from the musicological literature. 


\section{CHORDS CONVEYING NOSTALGIA}

In the recent study by Lahdelma and Eerola (2014), an empirical experiment was carried out where participants $(N=269)$ evaluated pre-selected chords on a 9-item scale of given (closed set of terms) emotional dimensions spanning both basic (e.g., happiness and sadness) as well as mixed emotions (e.g., nostalgia/longing). The emotion terms the experimenters selected were based on previous empirical research (e.g., Juslin et al., 2008; Zentner et al., 2008). Fourteen different chords (major, minor, diminished, and augmented triads and dominant, major and minor seventh chords with inversions) were played with two distinct timbres (piano and strings). The triad chords were those of $\mathrm{C}$ major $(\mathrm{C}), \mathrm{C}$ minor $(\mathrm{Cm}), \mathrm{C}$ diminished $\left(\mathrm{C}^{\circ}\right)$ and $\mathrm{C}$ augmented $(\mathrm{C}+)$. The seventh chords were those of $\mathrm{C}$ dominant seventh (C7), $\mathrm{C}$ minor seventh (Cm7) and $\mathrm{C}$ major seventh (Cmaj7), all played in their root positions and third inversions respectively. The results indicated that the minor triad, the minor seventh, and especially the major seventh chords were quite effective at conveying the complex emotional quality of nostalgia. Of the single chords, the minor triad's root position and the major seventh's third inversion scored highest on nostalgia. When played in the string timbre, the modal nostalgia rating of both the minor triad and the major seventh chord was 4 ("Quite a bit") on the 5-point Likert scale, in each chord position. That is, a typical listener perceived "quite a bit" of nostalgia in these particular chords, with the major seventh chord's third inversion conveying the emotion of nostalgia more effectively than its root position. Intriguingly, the major seventh chord's third inversion's mean rating was highest on the dimension of nostalgia of all the given dimensions when played on the strings, implying that this particular chord conveys nostalgia par excellence. While the experimenter-selected, closed set of dimensions may have guided the participants' evaluations to some extent, this drawback is common to all methodologies that rely on verbal methods (Zentner \& Eerola, 2010). The strength of the original research protocol by Lahdelma and Eerola (2014) lies in the fact that the emotional dimensions that were utilized have been used in a large variety of past studies of emotional expression in music (e.g., Juslin \& Laukka, 2004; Juslin et al., 2008). Moreover, with the original experiment's Likert scale there was always the possibility to rate a chord very low (the lowest position on the Likert scale was "very slightly or not at all") if it absolutely did not seem to fit a certain dimension-- a possibility the participants also clearly used if we take, for example, the "happiness" of the augmented triad.

As for nostalgia/longing, this dimension was quite exclusively used for the chords of the minor triad, the minor seventh and especially the major seventh, making it an intriguing and tangible case to investigate further. As the aim of their study was to explore whether single chords are able to convey distinct emotions, Lahdelma and Eerola (2014) did not pursue any theoretical explanations of their observations, despite the plain need for a theoretical rationale to account for complex emotions such as nostalgia/longing.

\section{EXPLANATIONS FOR NOSTALGIA CONVEYED BY CHORDS}

As a way of describing how single chords might convey the complex emotion of nostalgia, we consider cultural and music-theoretical explanations, as well as their possible interactions. In addition to these explanations, we outline a probable physical mapping that underlies the proposed explanations. Our candidate explanations are (1) learning, (2) intrinsic emotional connotations arising from tonal relations, and (3) clashing conventions. We will describe these next in detail.

\section{Premise: Mapping Between Physical Properties and Affect States}

Connections between the emotional expressiveness of physical sounds and underlying physiological states have been posited at least since the work of Spencer (1857), implying that different emotions actually cause physiological changes that alter vocal expression. This physiological state explanation (Scherer, 1986) accounts for emotions expressed in speech, which in turn bears on musically communicated emotions as well. Juslin and Laukka (2003) have shown that there are strong parallels between vocal expression and musical expression of basic emotions (e.g., happiness and sadness). Most of these connections relate to amplitude, tempo/speech rate, and timbre, but there are also links to be found between intervals and emotional states. For instance, according to Bowling, Gill, Choi, Prinz, and Purves (2010), the spectra of major intervals are more similar to spectra found in excited speech, whereas the spectra of particular minor intervals are more similar to the spectra of subdued speech. Also, Curtis and Bharucha (2010) propose that 
the minor third occurs in the pitch contour of speech conveying sadness, as well as in music. Similarly, Huron (2008) has shown that small melodic intervals are more prevalent in sad than happy music, and demonstrated that this feature is directly related to the possible interval sizes available in scales (Huron \& Davis, 2012). In general, connections between certain intervals (both vertical and horizontal) and emotional expression have been found in several empirical studies (e.g., Costa, Bitti, \& Bonfiglioli, 2000; Cousineau, McDermott, \& Peretz, 2012; Krantz, Merker, \& Madison, 2004; Maher, 1980; Oelmann \& Laeng, 2009), showing typically that the minor and the major second, and the minor and the major seventh are the most displeasing intervals, possessing the most negative valence (intrinsic attractiveness vs. aversiveness), with the major seventh also correlating with sadness ratings (e.g., Krantz et al., 2004).

The underlying reason why these particular intervals seem to be effective at conveying negative valence may be due to the amount of dissonance they contain (cf. Hutchinson \& Knopoff, 1979; Kameoka \& Kuriyagawa, 1969; Malmberg, 1918). Zentner and Kagan (1996) propose that the preferential bias for consonance could be innate, basing this view on their findings that even infants are biologically prepared to treat consonance as more pleasant than dissonance. This aversion to dissonance has a biological substrate. As Peretz (2010) puts it:

Sensory dissonance can be created by two simultaneous tones one or two semitones apart. This combination creates beating at the level of the basilar membrane in the inner ear. The overlap in vibration patterns compromises the resolution of pitches of different frequency on the basilar membrane, leading to the perception of roughness. (p. 108)

However, McDermott et al. (2010) curiously attribute harmonicity as a more important factor in aversion to dissonance than roughness, with harmonicity meaning "how closely the spectral frequencies of a sound correspond to a harmonic series" (Parncutt, 1989, p. 172). For example, a major triad has high harmonicity as it is "more similar to the harmonic series as it exists among the audible partials of everyday harmonic complex tones such as voiced speech sounds" (Parncutt, 2012, p. 130). Moreover, as Johnson-Laird, Kang, and Leong (2012) point out, roughness in itself fails to predict the relative dissonance of, for example, common triad chords. It has been proposed that dissonance is in fact based on a combination of roughness, harmonicity and familiarity (Parncutt, 2012). These affective parallels between certain intervals and broad affects are likely to have an impact on the emotional perception of single chords too, as we will later demonstrate.

\section{1) Learning}

Learning seems to play an important role in the perception of emotions conveyed by vertical harmonies. Peretz (2010) suggests that "among adults, there is robust evidence that emotional responses are modulated by experience" (p. 103) and that "the unconscious affective influence of prior exposure to music may account for a vast array of phenomena" (p. 104). These phenomena include, for instance, the emotional distinction between the major and minor mode. These, in turn, are probably shaped by the underlying physical and physiological states mentioned in the previous section. Previous research indicates that tempo is mastered earlier than mode to judge the emotional tone conveyed by music (Dalla Bella, Peretz, Rousseau, \& Gosselin, 2001). This suggests that the major-happy/minor-sad association is indeed learned, and that the major/minor triads can hence be seen in a Peircean sense as "indices" of happiness/joy and melancholy/sadness - at least in Western music. The origin of the major-happy/minor-sad association dates back to probably the $14^{\text {th }}$ century and is still disputed (see for example Crowder, 1984; Parncutt, 2012, 2014). One recent study interestingly suggests that newborn infants' auditory systems are actually sensitive to Western music chord categories (Virtala, Huotilainen, Partanen, Fellman, \& Tervaniemi, 2013). However, Parncutt (2012) points out that the major-happy/minor-sad association may be absent in historical or non-Western traditions that are not based on triadic harmony, but also suggests that the basis of the major/minor emotional distinction is not necessarily only in arbitrary associations. Similar suggestions have been made by, for example, Crowder (1984). Parncutt $(2012,2014)$ sees that the major mode is perceived as a standard form as opposed to the minor mode because music in major keys is more common in Western music. According to Parncutt, this is due to the major triad's closer similarity to the harmonic series and that the major triad is in this sense more consonant than the minor triad - a view suggested also by, for example, Helmholtz (1895/1980), Schenker (1906), and Schoenberg (1922/1990). Whatever the origin of the major/minor emotion convention might be, according to previous empirical 
research (e.g., Lahdelma \& Eerola, 2014; McDermott et al., 2010; Pallesen et al., 2005), the major triad is indisputably an effective index of happiness/joy, while the minor triad is an index of melancholy/sadness in Western culture, even though isolated major/minor triads' perception can, of course, also be affected by pitch height, loudness and timbre (Parncutt, 2012).

Contemplating the issue from a historical point of view, Tagg and Clarida (2003) suggest that

the nineteenth-century Central European tonal system put minor modes de facto into the cultural position of archaisms. Ousted by the then 'more modern' major key, minor could acquire general connotations of oldness and the past and...lead associations...into nostalgia, quietude and sadness. (p. 313)

As for the effective mediation of these connotations, Tagg and Clarida point out that early film music grounded its semantic practices on European classical and romantic music. According to Cohen (2010) "films provide a major source for transmission of a culture's musical conventions" (p. 899). The emotional connotations that Tagg and Clarida attribute to the minor mode seem also to be present in the isolated minor triad chord as well (Lahdelma \& Eerola, 2014).

However, cultural conventions effectively mediated by film music is not the only possible explanation as to how the minor triad is able to convey nostalgia, in addition to melancholy and sadness. Juslin et al. (2013) propose that as a music-evoked emotion, nostalgia can also be a by-product of sadness. Also, Wildschut et al. (2006) and Sedikides et al. (2008) suggest that sadness and negative mood is often a trigger of nostalgia. A recent study by Taruffi and Koelsch (2014) suggests that nostalgia is actually the most frequent emotion evoked by sad music. This could be surmised to be an effect of episodic memory, in the sense that the melancholic and sad stimuli trigger episodic memories and hence nostalgia. Juslin et al. (2013), for example, explain unfamiliar music's ability to arouse nostalgia in listeners with this particular mechanism. However, according to the empirical data of Lahdelma and Eerola (2014), the nostalgic minor seventh and major seventh chords scored relatively high on the dimension of valence, and the minor seventh chord in turn scored quite low on the dimension of melancholy/sadness. Also, this trigger could actually be any aspect of music, and pinning it down to particular chords is a tall order. Another crucial counterargument to this explanation is how in the above-mentioned data the augmented and the diminished chords conveyed melancholy/sadness effectively but conveyed nostalgia/longing rather ineffectively. These results imply that the mechanism of episodic memory by itself does not offer a comprehensive explanation of how single chords are able to convey nostalgia.

In sum, learning seems to account for the broad connotations of major-minor triads (happy and sad), but whether this goes further to encompass more complex emotions such as nostalgia is uncertain.

It could be argued that musical conventions utilize certain chords (e.g., major sevenths) in conveying nostalgia, longing and wistfulness in film music (e.g., Ennio Morricone's Canzone della Nostalgia [Song of Nostalgia] from the film And Agnes Chose to Die [1976] and Gustav Mahler's distinctively longing music used in, for instance, Luchino Visconti's Death in Venice [1971]), as well as in both classical and popular music. There are quite clear-cut examples to be found in musicological literature to illustrate the use of major seventh chords with specific regard to the expression of nostalgia and longing. According to Fleming and Veinus (1958), the major seventh chord in the final duet ( $O$ terra addio) from the opera Aida "...creates an almost unbearable tension that perfectly expresses the infinite longing of the doomed lovers on the brink of eternity" (p. 67). Curiously, Cooke (1959) also notes that in this very same closing duet the major seventh chord denotes a "violent longing for joy" (p 75). Cooke (1959) further discusses the major seventh chord and suggests that Delius "...found it most useful, added to the major triad, pianissimo, unresolved, to express his obsessive nostalgia (the opening chord of On hearing the First Cuckoo in Spring)" (p. 76), and that Sibelius "found it an admirable note to add to the tonic triad, fortissimo" at the end of his Seventh Symphony to convey a mood of intense "aspiration" (p. 76). Leonard Bernstein's (1976) analysis of Mahler's Fifth Symphony's Adagietto in his classic fourth Harvard lecture is also of interest here. When discussing the piece's "affective ambiguity," Bernstein (1976) specifically mentions the appoggiatura "E" in the melody being an "unresolved tug-at-the-heart" (p. 199) in the context of the F major chord. It is noteworthy how, in many sources, this "affective ambiguity" with regard to the Mahler Symphony and its Adagietto is interpreted as particularly "nostalgic" in nature. In the BBC Proms (2011) foreword with regard to the Fifth Symphony this connection is clear: "Written whilst he was recuperating from a sudden life-threatening illness, it is full of nostalgia" (BBC, 2011). Also, in the Mahler Companion (2002), edited by Donald Mitchell and Andrew Nicholson, this association is conspicuous and 
rather remarkable: “...Visconti used the Adagietto in his film of Mann's Death in Venice (1971) as sonorous symbol of Aschenbach's nostalgia, frustrated passion, and hopeless longing...” (p. 310). With regard to pop music this linkage is also clear; for example, Everett (2008) discusses the major seventh chord sounding "nostalgic" and "wistful" (p. 200), while drawing concrete examples from songs like Old Friends by Simon and Garfunkel, and Old Cape Cod by Patti Page.

\section{2) Intrinsic Emotional Meaning Built Into Tonality}

Learning this connotation through extensive exposure to it in various musical styles and contexts is, however, only one possible explanation to account for nostalgia and longing being conveyed by the major seventh chord. In addition to the theory of learning, we contemplate the possibility of nostalgic emotional meaning arising from what Juslin (2013) calls intrinsic coding. Juslin (2013) explains intrinsic coding to involve internal syntactic relationships within the music itself. He also points out that intrinsic sources of musical expression in music have rarely been investigated, but that they are unlikely to express specific emotions by themselves. This explanation could theoretically account for the major seventh chord's ability to convey nostalgia, stemming from the tension of pitch relationships among tones that is present in tonality. Cooke (1959) explains the nostalgic or longing effect of the major seventh chord by proposing that its

tension in relation to the triad is a semitonal one upwards the tonic, so that when it is exposed in relation to the major triad, the 'longing for pleasure' it evokes is so violent as to be almost painful. It is, moreover, a longing for pleasure in a context of finality, aiming to the tonic...(p. 76)

Previous empirical experiments have offered Cooke's semantic views only limited support (Kaminska \& Woolf, 2000), or no corroboration at all (Gabriel, 1978). However, none of these experiments tested Cooke's views on the emotional effects of vertical harmony per se. Like Cooke, Huron (2006) also takes notice of the leading tone's distinctive quale, attributing it a "strong sense of precariousness or instability mixed with some urgency and accompanied by feelings of yearning or aspiring upward" (p. 144). As Huron (2006) suggests: "the feelings that precede highly expected events are quite distinctive" (p. 306). The leading tone's resolution upwards to the tonic is a highly expected outcome in Western harmony according to an intriguing anecdote (see Kelly, 2001), composer Maurice Ravel had actually been eliminated from the Prix de Rome scholarship for ending a piece with a chord containing the major seventh.

Cooke's semantic theory (1959) implies that chords do not create emotional meaning solely by perceived consonance/dissonance, as is suggested by Terhardt's (1974) view that listeners evaluate single, isolated chords on the basis of sensory consonance and (horizontal) harmony through a higher cognitive process. Also, Huron (2006) proposes that "the ability of listeners to imagine a single tone as serving different tonal functions indicates that scale degrees are cognitive rather than perceptual phenomena" ( $\mathrm{p}$. 143), adding that scale degree is "how minds interpret physically sounding tones, not how tones are in the world" (p. 143). Cooke's line of thinking coincides also with the view of Tramo, Cariani, Delgutte, and Braida (2001), who suggest that "a listener's implicit (or explicit) knowledge about harmony in the horizontal dimension bears on harmony perception in the vertical dimension" (p. 96), suggesting that the perception of vertical and horizontal harmony are not necessarily that far apart. Krumhansl (1990) proposes that "a tonal context designates one particular tone as most central" and that "the other tones all have functions specified with respect to this tone" (p. 18). Tillman, Bharucha, and Bigand (2000) suggest that adults with little or no musical training also possess this implicit knowledge. Although Cooke's notion is clearly rooted in tonality and therefore pertinent to horizontal harmony (Cooke actually proposes that the longing effect of the major seventh is present when added specifically to the tonic triad), the findings of Lahdelma and Eerola (2014) suggest this effect is present in the single, isolated major seventh chord as well, without a tonal or musical context: the major seventh was rated as the most nostalgic chord alongside the minor triad in their data. According to Parncutt (2011), in major/minor tonality a chord must normally progress to its dominant and back before establishing a proper tonality. Thus, a single isolated chord most likely cannot function as a tonic, or to be precise, in this case the major seventh chord's triad "base" cannot function as a "tonic" to the seventh tone within the chord. 
The "iconic" (in the Peircean sense) yearning stemming from tonal relations (intrinsic coding) in single chords is problematic for a number of other reasons, too. First, Juslin (2013) proposes that intrinsic coding may require a relatively long timespan (structures alternating in tension and release in a broader musical context) to be effective. Hence, a stimulus as short as a single chord would not count as the most probable candidate for being able to convey emotion by intrinsic coding, unless the chord's emotional meaning is actually rather effectively acquired from exposure to tonality (in this case the emotion perception of the major seventh chord would actually be a mixture of the explanations of learning and intrinsic emotional connotations arising from tonal relations, a possibility discussed further in the discussion section).

Second, if the additional tones would indeed create "iconic" tension in relation to the major triad as Cooke (1959) suggests, then why, one might ask, is the dominant seventh chord conveying this particular emotion quite ineffectively (cf. Lahdelma \& Eerola, 2014)? After all, Cooke remarks that just as the major seventh is longing upwards to the tonic, the minor seventh is accordingly drawn downwards to the dominant. If the major seventh chord's nostalgia stems from "tension" and the leading tone's aspiration to the tonic, it is hardly corroborating his theory that in the dominant seventh's case, these same attributes do not seem to convey nostalgia in any effective way: it is noteworthy that the dominant seventh chord conveyed considerably less nostalgia/longing than the major seventh chord, but scored higher on the dimensions of interest/expectancy and tension (Lahdelma \& Eerola, 2014). Cooke (1959) even suggests that the dominant seventh interval in relation to the major triad expresses "a gentle mournful feeling" ( $p$. 74). In fact, according to the empirical results of Lahdelma and Eerola (2014), Cooke's view of the dominant seventh chord's "sad, empty sound" (p. 74) obtained no empirical support, since it scored relatively high on the dimension of valence but low on the dimension of melancholy/sadness. This also hints at the possibility that, accordingly, the minor seventh chord conveys nostalgia effectively because of its minor triad "base" rather than because of the minor seventh tone's longing to the sixth or to the dominant, as implied by Cooke's line of thinking.

Finally, the difference in the perceived amount of nostalgia/longing in the two inversions of the major seventh chord is also noteworthy (Lahdelma \& Eerola, 2014). The higher amount of perceived nostalgia/longing in the third inversion including the interval of the minor second, as opposed to the root position including the interval of the major seventh, hints at the possibility that the presence of the most dissonant interval of the minor second (cf. Hutchinson \& Knopoff, 1979; Kameoka \& Kuriyagawa, 1969; Malmberg, 1918) creates more nostalgia in the chord than the presence of the slightly less dissonant major seventh. Both chord positions, however, clearly convey the emotion of nostalgia, and the difference in chord position might be stemming from a difference in register (in the root position the seventh tone is an octave higher than in the third inversion). As none of the presented explanations seem to comprehensively account for the major seventh chord's ability to convey nostalgia, we propose a new theory as the third explanation.

\section{3) Clashing Conventions}

\section{According to Meyer (1956)}

States of calm contentment and gentle joy are taken to be the normal human emotional states and are hence associated with the more normative musical progressions, i.e., the diatonic melodies of the major mode and the regular progressions of major harmony. ( $\mathrm{p}$. 227)

These same attributes could well describe the isolated major triad as well, as it is highly effective at conveying happiness/joy on the basis of previous empirical research (e.g., Lahdelma \& Eerola, 2014; McDermott et al., 2010; Pallesen et al., 2005). The major triad is also the most common chord in Western harmony (Huron, 2006). In light of the major triad's conventional role as the "norm" of positive valence (e.g., Meyer, 1956; Parncutt, 2012), one could consider how additional tones may alter its valence and emotional meaning, this time contemplated from another perspective than Cooke's (1959) suggestion of "iconic" meaning arising from tonal tension which attributes the leading tone a special "affective quale" (cf. Huron, 2006) in relation to the tonic. As mentioned in the premises section, the minor second and the major seventh intervals have been show empirically to score high on sadness and negative valence. Hence, these particular intervals could be seen as "obscuring" the positive valence of the triad, although not 
altogether "obliterating" it. In other words, the "clashing" of two highly conventionalized indices (the major triad as an index of happiness/joy, and the dissonant intervals of the minor second and the major seventh as indices of negative valence and melancholy/sadness) could create new emotional meaning that is perceived as the complex emotion of nostalgia. In other words, both positive and negative emotions might be conveyed by the major seventh chord concurrently, creating "affective dissonance" which in turn is perceived as the bittersweet emotion of nostalgia. The proposed clashing conventions explanation is compatible with many analytical descriptions of nostalgia that combine happiness/joy and melancholy/sadness. On an aesthetic level, several definitions of nostalgia have intriguing parallels with this explanation. Batcho (2007), for example, suggests that conflict is inherent in the nostalgic experience, and that "theories that emphasize the bittersweet quality of nostalgic sentiment attribute the bitter side to the realization of the irretrievability of the past and the accompanying sense of irrecoverable loss" (p. 375). Nostalgia is also seen as a yearning for an idealized past (Hirsch, 1992). It is interesting to speculate about the major triad being the "idealized" item, the norm of positive valence which is altered or "obscured" by the sad and negative intervals of the minor second or the major seventh, which are also the sharpest dissonances (Hutchinson \& Knopoff, 1979; Kameoka \& Kuriyagawa, 1969; Malmberg, 1918). Also, Werman (1977) sees nostalgia as "wistful pleasure, a joy tinged with sadness" (p. 393). Again, the analogy of the major triad representing happiness/joy, while the minor second and its inversion--the major seventh-represent the tinge of melancholy/sadness, is striking. Interestingly, the major seventh chord is also the clashing of the major and minor triads (C-E-G, major; E-G-B, minor), and at this point it is not possible to rule out this inherent feature as a contributor in conveying the chord's nostalgic connotation in addition to the two sad and negative sounding intervals clashing with the major triad.

According to Juslin (2013), listeners' interpretations of emotions in music tend to gravitate towards basic categories and Juslin suggests that basic emotions (e.g., happiness, sadness) are at the core of human emotions. In the major seventh chord's case, one could argue that because these basic emotions are so strong, they might create more subtle nuances and convey mixed emotions when overlapping or being present concurrently. Juslin (2013) proposes that according to many emotion researchers, "mixed emotions" are founded on basic emotions that involve "blends" of emotions. Hunter, Schellenberg, and Schimmack (2010) suggest that there is "empirical evidence demonstrating that positive and negative affect (i.e., corresponding to opposite poles of the putative valence dimension) are activated simultaneously in some circumstances" (p. 48), but remark that not both emotions are felt strongly when being present simultaneously. Instead, they propose that "one response (i.e., the dominant response; e.g., sadness) is usually stronger than the semantically opposite response (i.e., the conflicting response; e.g., happiness)" (p. 48). Interestingly, in the case of the major seventh chord, listeners perceived more melancholy/sadness than happiness/joy in the chord, and the mixed emotion of nostalgia/longing seemed to capture the chord's affective quality better than either of the two aforementioned dimensions representing basic emotions (Lahdelma \& Eerola, 2014).

The clashing conventions explanation needs certain premises to have thorough explanatory power, however. If the concurrence of the major triad and a minor second/major seventh would always create nostalgia, a dissonant chord like the major triad with an added augmented fifth (C-E-G-G\#) should sound "nostalgic" as well. Intuitively this is, of course, not the case: instead of sounding nostalgic the chord is highly unstable due to its interval distribution, namely the concurrence of two major thirds (C-E and E-G\#). Meyer (1956) proposes that augmented and diminished triads produce ambiguity because of their "intervallic equidistance" (p. 164), an even spacing between the tones in the chord. He points out that these chords are identified with affectivity and are often "used to express intense emotion, apprehension, and anxiety" (p. 164). However, this effect can perhaps be better explained with the concept of tonal dissonance. Johnson-Laird et al. (2012) propose that the perception of dissonance in music can be explained with a dual-process theory of dissonance. They suggest that dissonance

results from a combination of sensory and tonal dissonance, where 'sensory' dissonance arises...in particular from roughness (i.e., the rapid beating of partials), and 'tonal' dissonance is a consequence of high-level cognitive processes that rely on a tacit knowledge of the principles of tonality. (p. 24)

They propose that tonal dissonance depends on the scales in which the chords can occur: 
Chords occurring in a major scale should be less dissonant than chords occurring only in a minor scale, which in turn should be less dissonant than chords occurring in neither sort of scale. (p. 24)

Finally, they suggest that

chords that are consistent with a major triad are more consonant than chords that are not consistent with a major triad...hence, a chord of a seventh, such as GBDF, is consistent with a major triad (GBD) because the seventh, F, occurs in a major scale in which the triad also occurs (the scale of $\mathrm{C}$ major), whereas a chord, such as GBDEb, is not consistent with a major triad because the added note does not occur in a major scale containing the major triad. (p. 24)

Obviously, these principles should then logically bear on the emotion perception of single chords, as well as on their perceived amount of dissonance.

The low amount of tonal dissonance is an essential premise for the clashing conventions explanation. In relation to a $\mathrm{C}$ major triad there are only three possible minor seconds (the major sevenths can be regarded as the inversions of the minor seconds) that would create minimal tonal dissonance (those minor seconds that are present in major diatonic scales): E-F, B-C and F\#-G. So, this narrows the possible combinations of the concurrence of a major triad and a minor second (and their respective inversions) within diatonic major scale contexts to the following sets of tones: C-E-G-B (a major triad with an added major seventh, present in C major and in G major), C-E-F-G (a major triad with an added fourth, present in $\mathrm{C}$ major and in $\mathrm{F}$ major), and the unusual chord of C-E-F\#-G (a major triad with an added augmented fourth, present theoretically in $\mathrm{G}$ major). The major triad with an added fourth contains more roughness than the major seventh chord (Figure 1), yet the added fourth chord would intuitively seem to convey less nostalgia than the major seventh chord. We propose that this might be explained by the difference in the amount of harmonicity the chords contain: the major triad with an added fourth has higher harmonicity than the major seventh chord (Figure 2). As $\mathrm{F}$ can be seen as a missing fundamental of the $\mathrm{C}$ major triad (Parncutt, 2012), B is arguably simply more "alien" in the C major triad's context than F. It can be argued that a larger amount of harmonicity could indeed create more positive valence as, according to Parncutt (2012), it also creates more consonance. According to a similar logic, the major seventh chord could in turn create slightly more "negative valence" and hence nostalgia than the added fourth chord, as in addition to the clashing of the affective indices (major triad as an index of happiness, the minor second/major seventh of negative valence), B is a more distant tone within a C major triad context than F. In Parncutt's (1993) experiment, listeners rated how well probe tones went with preceding chords. In regard to the major triad, the probe tone of the perfect fourth was rated to fit the major triad better than the major seventh.

The same holds true for the major triad with an added augmented fourth: it is not rough (Figure 1) and has low tonal dissonance, but what makes it "un-nostalgic" is the high amount of harmonicity it contains (Figure 2). It is also intriguing to have a look at the dominant seventh triad as well, as it could also be seen as an example of a "clashing convention": in its root position (C-E-G-Bb) it is a major triad (C-EG) with an added minor third (G-Bb). According to Cooke (1959) this clash is what gives the chord its "sad and empty sound" (p. 74), stemming from the "undermining of the normal joyful feeling of the major triad supporting it" (p. 74), a view that obtained no empirical support in the study of Lahdelma and Eerola (2014). The chord has a strikingly low amount of roughness (Figure 1), has no tonal dissonance and contains a high amount of harmonicity (Figure 2). Also, Parncutt (2014) suggests that the dominant seventh "looks like a part of the harmonic series," as 'the difference between the 'harmonic' ('natural', 'septimal') minor 7 th $(7: 4,969$ cents) and the minor seventh in 12-tone equal temperament (1000 cents) lies within the tuning tolerance of the auditory system for recognizing harmonic patterns" (p. 328). Again, we surmise that the higher amount of harmonicity results in a lower amount of perceived nostalgia: the dominant seventh was not rated as nostalgic according to the data of Lahdelma and Eerola (2014). Interestingly, the moderately nostalgic minor seventh chord (Lahdelma \& Eerola, 2014) also has only a medium amount of harmonicity (Figure 2). The nostalgia predictions of selected chords and the amount of tonal dissonance, harmonicity, and roughness they contain can be seen in Table 1. 


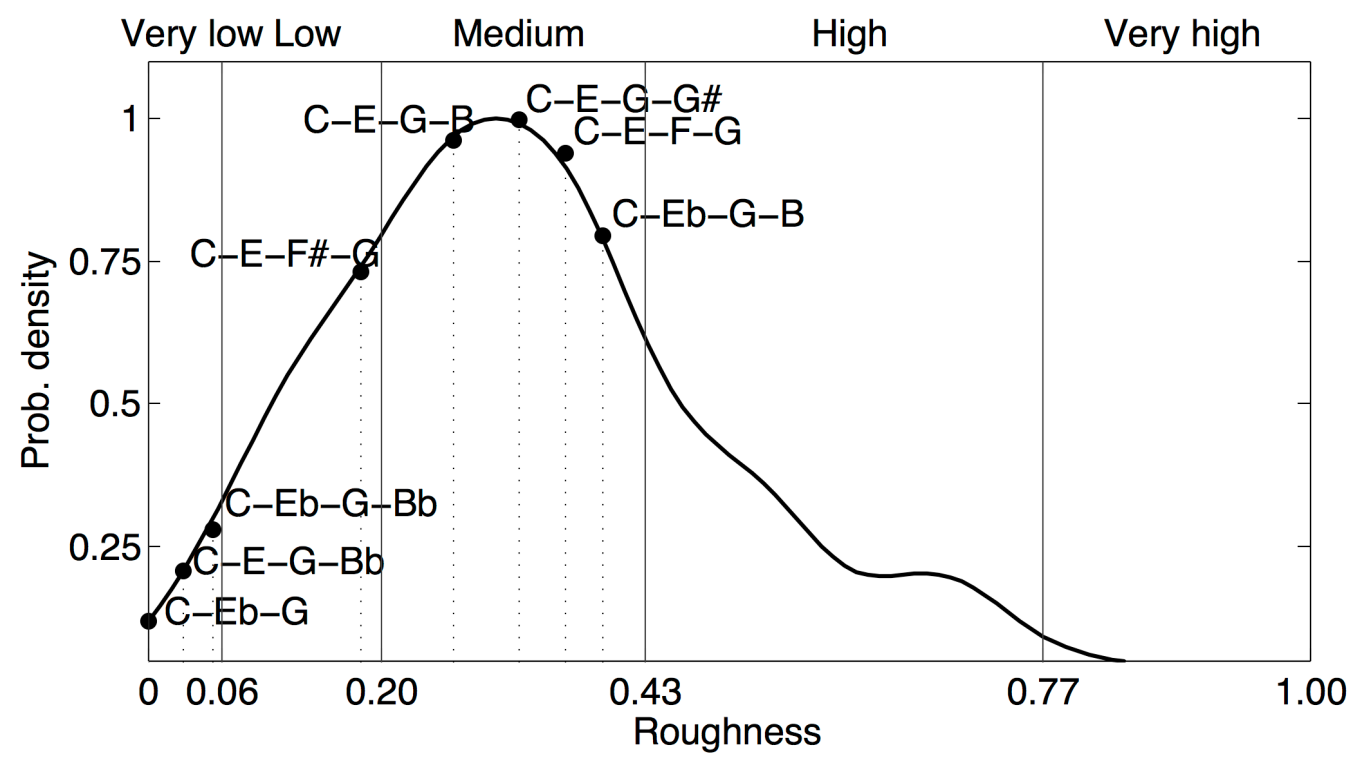

Fig. 1. Roughness as a probability density of all four-tone chords within an octave, with eight example chords highlighted.

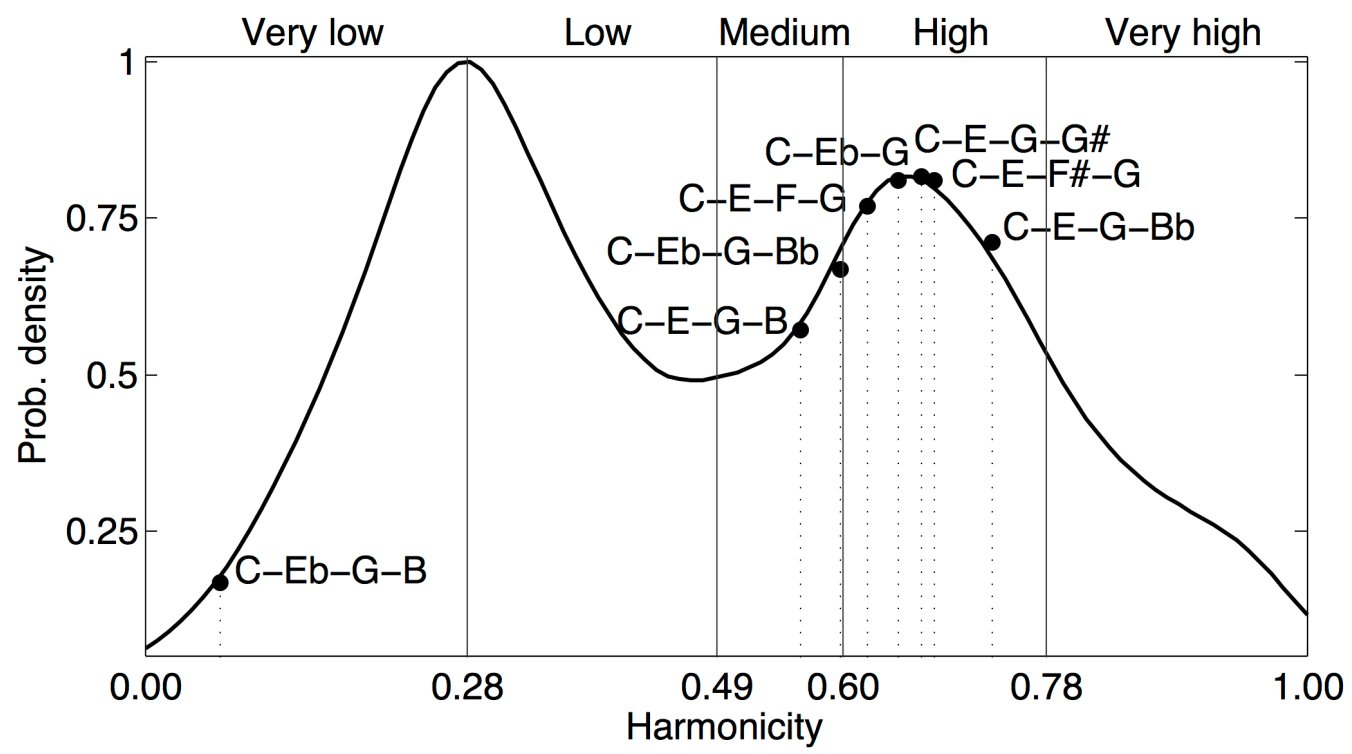

Fig. 2. Harmonicity as a probability density of all four-tone chords within an octave, with eight example chords highlighted.

The psychoacoustic predictions were estimated by first creating all possible four-tone chords within an octave (resulting in 536 separate chords, discounting the inversions). These chords (and one three-tone chord, $\mathrm{C}-\mathrm{Eb}-\mathrm{G}$, for a reference) were implemented as a combination of four equally loud fundamentals that were transformed into complex sounds with Shepard spectra using MIDI toolbox (Eerola \& Toiviainen, 2003). These sounds were in turn analyzed with MIR toolbox (version 1.5) for harmonicity (Jensen, 1999) and roughness (Vassilakis, 2001). Since this analysis yielded a broad range of values which by themselves are not meaningful unless used in a context, the output was set to vary between 0 and 1 and the feature histograms were converted into probability densities. From these, the quantiles were used to simplify and express the values from Very low to Very high (see Figure 1 and 2 for the quantile boundaries and individual chord values, whereas Table 1 displays only the value categories). In Table 1 , the tonal dissonances are empirically reported values taken from Johnson-Laird et al. (2012). 
Table 1. Theoretical predictions of nostalgia and musical/psychoacoustic attributes of selected chords (in middle register voicings with Shepard tones).

\begin{tabular}{llllll}
\hline No. & Chord & $\begin{array}{l}\text { Nostalgia } \\
\text { prediction }\end{array}$ & $\begin{array}{l}\text { Tonal } \\
\text { dissonance }\end{array}$ & Harmonicity & Roughness \\
\hline 1 & C-E-G-B & High & Low & Medium & Medium \\
2 & C-E-F-G & Moderate & Low & High & Medium \\
3 & C-E-G-G\# & Low & High & High & Medium \\
4 & C-Eb-G-B & Low & Medium & Very low & Medium \\
5 & C-Eb-G-Bb & Moderate & Low & Medium & Very low \\
6 & C-Eb-G & High & Low & High & Very low \\
7 & C-E-G-Bb & Low & Low & High & Very low \\
8 & C-E-F\#-G & Low & Low & High & Low \\
\hline
\end{tabular}

\section{SUGGESTIONS FOR EMPIRICAL EXPERIMENTS}

No direct empirical tests have yet been conducted on the issue of how vertical harmony is able to convey nostalgia. To disentangle which of the current study's proposed explanations would prevail and whether some of these explanations would be more valid in the case of certain chords than others, further empirical experiments are needed. Also, a broad chord palette including chords that were not tested in the study of Lahdelma and Eerola (2014) could help in further investigating the question of vertical harmony being able to convey nostalgia and longing. Cooke (1959), for example, proposes that the major sixth chord is able to create "pleasurable longing" (p. 69), and suggests that the dominant thirteenth chord has a "yearning" quality (p. 70). Tagg and Clarida (2003) propose that the added major ninth in minor triads embodies qualities of "the bitter-sweet, sad lonely and longing, at least in European classical (chiefly romantic) music, as well as in the idiom of traditional film music" (p. 577).

In the major seventh chord's case it is intriguing to test which of the proposed explanations (learning, intrinsic emotional meaning built into tonality or clashing conventions) might better explain its ability to convey nostalgia. Intuitively, a simple test of also playing the minor major seventh chord (C-EbG-B) to the participants could tell more about the validity of the "iconic" longing Cooke attributes to the leading tone - whether the leading tone indeed conveys longing iconically in vertical harmony (single chords) as well. Cooke's theory would certainly imply that the leading tone would be longing to the tonic in the minor major seventh chord as well: after all, if horizontal harmony would indeed affect chord perception in this way, the leading or aspiring "quale" of the seventh tone would surely be learned from the harmonic or (ascending) melodic minor scale's leading tone as well as from the major scale. Cooke (1959) actually makes clear that he is omitting certain chords' emotional connotations (for example the "minortriad-plus-major-sixth") and although not mentioning it directly, also the minor major seventh chord, in order to confine his investigation to "basic elements" of what he calls "the language of music" (p. 72). From a present-day scholar's view this looks somewhat like an attempt on Cooke's behalf not to contradict 
himself. The authors surmise that the minor major seventh chord would not convey nostalgia in any effective way because of the chord's tonal dissonance; Johnson-Laird et al. (2012) suggest that in principle

the chords of major sevenths (e.g., CEGB), minor sevenths (e.g., DFAC), sevenths (e.g., GBDF), and half-diminished sevenths (e.g., BDFA) occur in major scales and so they should be less dissonant than minor triads with major sevenths, e.g., ACEG\#, augmented triads with a major sevenths (e.g., CEG\#B) and diminished sevenths (e.g. AbFBD), which occur only in minor scales. (p. 24)

Also, the minor major seventh has a considerably higher amount of roughness than the major seventh chord (Figure 1), and has a very low amount of harmonicity (Figure 2). The minor major seventh chord's higher tonal dissonance, roughness and very low harmonicity seem to result in a rather "dark" and "sinister" voicing (technically also a clashing of the minor triad [C-Eb-G] and the major third [G-B], its emotional effect yet to be tested empirically). The chord is notorious for its use in, for example, Bernard Herrmann's film score for Alfred Hitchcock's classic horror-thriller Psycho (1960), and has been referred to as the "Hitchcock-chord" (Brown, 1994), underlining its ominous quality.

\section{DISCUSSION}

Our aim was to account for the empirical findings of Lahdelma and Eerola (2014), according to which certain single, isolated chords (minor triads, minor sevenths and especially major sevenths) are moderately effective at conveying the mixed, complex emotion of nostalgia. These results raise the question whether there is a possibility that nostalgia in music is not necessarily related only to episodic memory (extramusical connotations), but that it can be conveyed even by abstract, reduced stimuli such as single chords outside a musical and tonal context. To account for this finding, we outlined three different candidate explanations, including (1) learning, (2) intrinsic emotional connotations arising from tonal relations, and (3) clashing conventions. The question of which one of the outlined explanations bears the most explanatory power will have to be dealt with in further empirical experiments. It is clear, however, that the suitability of the explanations seem to depend also on the chord in question. We surmise that with the minor triad the learning theory could be the most probable explanation, as with a single triad there are no "intrinsic" meanings built into the chord, be it emotional connotations arising from tonal relations or the current authors' theory of clashing conventions. In addition to the minor mode (Tagg \& Clarida, 2003), the isolated minor triad also seems to be affiliated with sadness, melancholy, and also nostalgia. However, in addition to the learning perspective, there exists the possibility that psychoacoustics could affect the emotion perception of the minor triad as well, although the amount of roughness and harmonicity the minor triad contains is quite similar to that of the major triad if one considers a wide range of different sonorities. According to Parncutt (2014), the minor triad has a more ambiguous main pitch or root than the major triad; curiously, Tagg and Clarida (2003) suggest that in the emotion of longing there is also an inherent "uncertainty." Could this ambiguity of the root in the minor triad when compared to the major triad lead to associations of nostalgia and longing as well?

Nostalgia in itself is a culturally loaded and a quite ambiguous term, which can also be seen from the subtle differences between US and Finnish respondents in regard to this particular emotion (Lahdelma \& Eerola, 2014). Also, while the double term "nostalgia/longing" is somewhat robust, this umbrella term made it possible to gather the original data (Lahdelma \& Eerola, 2014) on a level that was delicate enough to showcase clear differences and underlying patterns, but was not so subtle so as to create too much diversion that could not have been interpreted in a meaningful way. As the music analytical examples from the literature show, there seems to be a definite connection between major seventh harmony and the emotions of "nostalgia" and "longing" along a music historical continuum. While subtle variations of the dimension may not be possible to capture with this umbrella term, it is quite remarkable that listeners did articulate and agree on this original label term as much as they did, especially with regard to the amount of nostalgia/longing perceived in the major seventh chord. The more subtle the variations under the umbrella term would be, the more individual differences between respondents we would be likely to find - with language and culture also affecting the responses more.

We see that in order to minimize the effect of possible emotion term confusions, future experiments could also distinguish between nostalgia and longing, to test if there are any differences to be found between these two emotion terms and whether one or the other actually captures the affective 
qualities of particular chords better. In regard to the study by Lahdelma and Eerola (2014), it is at this point unclear whether perceived nostalgia would hold up also with open questions, as opposed to pre-selected emotion terms, in the experiment design. However, according to their study a most interesting case in point concerning single chords' ability to convey the complex and mixed emotion of nostalgia is the major seventh chord. As the major seventh chord's third inversion's mean rating (when played in string timbre) was highest on the dimension of nostalgia of all the given emotion dimensions, it begs the question of how this particular chord is able to convey nostalgia so effectively. With string instruments the average ratings for the dimension of nostalgia/longing were somewhat higher than with the piano, suggesting the fact that the strings' soft timbre with a slow attack --as opposed to the piano's loud, sharp attack with a fast decay-convey this particular emotion more efficiently. Naturally, far from being a completely "fixed" and "lexical" meaning of nostalgia and longing, the major seventh chord is obviously subordinate to psychophysical (tempo, dynamics, phrasing) parameters as well, especially in actual music - just like the major and minor triads with regard to "happiness" and "sadness," respectively (Gabrielsson \& Lindström, 2010).

While it is not yet possible to entirely dismiss Cooke's (1959) theory of the major seventh chord's emotional properties arising from intrinsic tonal relations, this theory certainly has its flaws. Accordingly, the learning aspect also cannot be ruled out at this point, as there is a possibility that the major seventh chord's nostalgic connotation stems from learning and that this chord has simply been used in the context of music that the respondents somehow recall. In other words, the possibility of the major seventh chord being a case of evaluative conditioning just like the "indices" of the "happy" major and "sad" minor triads cannot be ruled out. According to this line of thinking, however, nearly all tonal chords should sound more or less "nostalgic," as they have been heard countless times in all kinds of settings. According to the empirical data of Lahdelma and Eerola (2014) this is clearly not the case, as only three chords were effective at conveying this particular emotion. In the minor seventh chord's case the question is whether its nostalgic connotation arises from the minor triad that is present in the chord, or whether the added minor seventh tone has something to do with its conveyed nostalgia. However, while the minor seventh was above average in its power to convey nostalgia, it was not as effective in this as the minor triad or the major seventh chord.

The possible interaction between the three proposed candidate explanations is also something to be considered. For example, by combining two of the proposed mechanisms, namely the (1) learning aspect with Cooke's (1959) proposal of certain chord's emotional properties arising from (2) tonal relations, the major seventh chord's nostalgic connotations might arise from a combination of these two. The leading tone's "longing" in relation to the major triad (also as an appoggiatura) might be learned from an extensive exposure to tonality. The dissonance in the major seventh chord, for example, can suggest a need to resolve without actually resolving. Interestingly, Toch (1948) proposes that imaginary motion gives meaning to audible symbols, referring to vertical harmony as "arrested motion" (p. 22). Thus, the thorough conditioning to voice leading processes could also be affecting the way an isolated chord is perceived. The minor major seventh chord's theoretical case of sounding rather "un-nostalgic," however, seems to somewhat challenge this view: while the major seventh in addition to the minor triad should accordingly suggest a need to resolve, the overall (isolated) sonority would probably not convey nostalgia or longing in any effective way because of the chord's relatively high (both sensory and tonal) dissonance, as discussed earlier. Consequently, the most salient difference between Cooke's and the current authors' theories is that, in sum, the clashing conventions explanation does not grant the leading tone any affective "quale" per se within the chord. Instead, we surmise that Cooke possibly misattributes the major seventh chord's nostalgia to the "longing" of the leading tone towards the tonic. However, the question of the role of a possible interaction between vertical and horizontal harmony with regard to nostalgia and longing in music should be investigated more thoroughly with future experiments.

It is interesting to compare the case of the virtually common-knowledge major triad-happy, minor triad-sad affective convention with some of the not so "well-conventionalized" seventh chords. The major and minor triads are extremely effective at conveying happiness/joy and melancholy/sadness (basic emotions) respectively, while the chord of the major seventh seems to be conveying the mixed emotion of nostalgia quite effectively, but slightly more ambiguously. As, from a psychological perspective, all perception depends on learning (Gibson, 1969), a crucial question is whether the effectively learned (by enculturation) two distinct "indices," i.e. the major triad as the index of happiness/joy, the minor second and its inversion as the index of melancholy/sadness might be able to create new, complex emotional meaning (nostalgia) when present concurrently in the major seventh chord. Or is the major seventh chord's 
nostalgic connotation learned per se, without any cognitive involvement in its emotional evaluation? The former view is in sharp contrast with the view of, for example, Terhardt (1974) who proposes that single isolated chords are judged on the basis of sensory consonance, and that there is nothing cognitive involved in this process (as opposed to the perception of horizontal harmony). Nostalgia, however, is not necessarily only confined to cognitive rumination: as a parallel to real-life nostalgia, it is interesting to think about how, for example, odors are able to create nostalgia directly without cognitive appraisal (Hirsch, 1992). The raised question might be relevant not only to the study of music psychology and musicology, but also to the study of emotions in general.

Parncutt (2012) suggests that the consonance/dissonance of Western sonorities is based on a combination of roughness, harmonicity and familiarity. We surmise that the same could well hold true also with regard to single chords' emotional impact, as not only roughness, but also harmonicity and tonal dissonance seem to affect emotional evaluations of single chords. After all, as Parncutt and Hair (2011) aptly put it, "harmony is an experience that lies at the interface between sensation and emotion" (p. 161). The tentative measurements of roughness and harmonicity in single chords compared to the existing empirical results of Lahdelma and Eerola (2014) suggest that the emotion perception of single chords and certain chords' ability to convey the mixed, complex emotion of nostalgia might be a delicate combination of cultural conventions and psychoacoustic phenomena, as well as learning. To shed light on this fascinating and elusive question, more empirical experiments are needed.

\section{ACKNOWLEDGMENTS}

We would like to thank László Stachó and Stephan Thomas Vitas for advisory reading and constructive remarks.

\section{NOTES}

[1] Corresponding Author. University of Jyvaskyla, Department of Music, P.O. Box 35, (M), FI-40014 University of Jyvaskyla, Finland. Email: imre.d.lahdelma@student.jyu.fi

\section{REFERENCES}

Bakker, D.R., \& Martin, F.H. (2015). Musical chords and emotion: Major and minor triads are processed for emotion. Cognitive, Affective, \& Behavioral Neuroscience, 15, 15-31.

Barrett, F.S., Grimm, K.J., Robins, R.W., Wildschut, T., Sedikides, C., \& Janata, P. (2010). Music-evoked nostalgia: Affect, memory, and personality. American Psychological Association, 10, 309-403.

Batcho, K.I. (2007). Nostalgia and the emotional tone and contents of song lyrics. The American Journal of Psychology, 120, 361-381.

BBC (2011). BBC Proms 2011: Mahler - Adagietto from Symphony No. 5 from Prom 69.

Retrieved from http://www.bbc.co.uk/programmes/p00k9fcg

Bernstein, L. (1976). The unanswered question: Six talks at Harvard. Cambridge, MA: Harvard University Press.

Bigand, E., Vieillard, S., Madurell, F., Marozeau, J., \& Dacquet, A. (2005). Multidimensional scaling of emotional responses to music: The effect of musical expertise and of the duration of the excerpts. Cognition and Emotion, 19, 1113-1139.

Bowling, D.L., Gill, K., Choi, J.D., Prinz, J. \& Purves, D. (2010). Major and minor music compared to excited and subdued speech. Journal of the Acoustical Society of America, 127, 491-503.

Boym, S. (2001). The future of nostalgia. New York: Basic Books. 
Brown, R.S. (1994). Overtones and undertones: Reading film music. Berkeley, CA: University of California Press.

Cheung, W., Wildschut, T., Sedikides, C., Hepper, E. G., Arndt, J., \& Vingerhoets, A. J. J. M. (2013). Back to the future: Nostalgia increases optimism. Personality and Social Psychology Bulletin, 39, 1484-1496.

Cohen, A.J. (2010). Music as a source of emotion in film. In Juslin, P. and Sloboda, J. (Eds.), Handbook of music and emotion: Theory, research, applications (pp. 879-908). Oxford: Oxford University Press.

Cooke, D. (1959). The language of music. Oxford: Oxford University Press.

Costa, M., Bitti, P. \& Bonfiglioli, L. (2000). Psychological connotations of harmonic musical intervals. Psychology of Music, 28, 4-22.

Cousineau, M., McDermott, J., \& Peretz, I. (2012). The basis of musical consonance as revealed by congenital amusia. Proceedings of the National Academy of Sciences, 109, 19858-19863.

Crowder, R. (1984). Perception of the major/minor distinction: I. Historical and theoretical foundations. Psychomusicology, 4, 3-10.

Curtis, M., \& Bharucha, J. (2010). The minor third communicates sadness in speech, mirroring its use in music. Emotion, 10(3), 335-348.

Dalla Bella, S., Peretz, I., Rousseau, L., \& Gosselin, N. (2001). A developmental study of the affective value of tempo and mode in music. Cognition, 80, B1-10.

Eerola, T. \& Toiviainen, P. (2003). MIDI toolbox: MATLAB tools for music research. Jyväskylä, Finland: University of Jyväskylä.

Eerola, T., Alluri, V., \& Ferrer, R. (2012). Timbre and affect dimensions: Evidence from affect and similarity ratings and acoustic correlates of isolated instrument sounds. Music Perception, 30(1), 49-70.

Everett, W. (2008). The foundations of rock: From "Blue Suede Shoes" to "Suite: Judy Blue Eyes". New York: Oxford University Press.

Filipic, S., Tillmann, B., \& Bigand, E. (2010). Judging familiarity and emotion from very brief musical excerpts. Psychonomic Bulletin \& Review, 17(3), 335-341.

Fleming, W., \& Veinus, A. (1958). Understanding music: Style, structure, and history. New York: Holt, Rinehart and Winston.

Freeman, J.W. (1992). The language of longing: Only at the final cadence does "Parsifal" resolve its musical question. Opera News, 56, 26-29.

Gabriel, C. (1978). An experimental study of Deryck Cooke's theory of music and meaning. Psychology of Music, 6, 13-20.

Gabrielsson, A., \& Lindström, E. (2010). The role of structure in the musical expression of emotions. In P. Juslin \& J. Sloboda (Eds.), Handbook of music and emotion: Theory, research, applications (pp. 393-414). Oxford, UK: Oxford University Press.

Gibson, E.J. (1969). Principles of perceptual learning and development. New York: Appleton-CenturyCrofts.

Helmholtz, H. (1895/1980). On the sensations of tone as a physiological basis for the theory of music (A. Ellis, Trans.). New York: Dover. 
Hindemith, P. (1942). The Craft of Musical Composition: Book 1-Theoretical Part (A. Mendel, Trans.). New York: Associated Music Publishers.

Hirsch, A.R. (1992). Nostalgia: A neuropsychiatric understanding. Advances in Consumer Research, 19, 390-395.

Hunter, P.G., Schellenberg, E.G., \& Schimmack, U. (2010). Feelings and perceptions of happiness and sadness induced by music: Similarities, differences, and mixed emotions. Psychology of Aesthetics, Creativity, and the Arts, 4(1), 47-56.

Huron, D. (2006). Sweet anticipation: Music and the psychology of expectation. Cambridge, MA: MIT Press.

Huron, D. (2008). A comparison of average pitch height and interval size in major- and minor-key themes: Evidence consistent with affect-related pitch prosody. Empirical Musicology Review, 3, 59-63.

Huron, D., \& Davis, M.J. (2012). The harmonic minor scale provides an optimum way of reducing average melodic interval size consistent with sad affect cues. Empirical Music Review, 7, 103-117.

Hutchinson, W., \& Knopoff, L. (1979). The significance of the acoustic component of consonance in Western triads. Journal of Musicological Research, 3, 5-22.

Janata, P.J., Tomic, S.T., \& Rakowski, S.K. (2007). Characterization of music-evoked autobiographical memories. Memory, 15, 845-860.

Jensen, T. (1999). Timbre Models of Musical Sounds, Rapport 99/7. Copenhagen, Denmark: University of Copenhagen.

Johnson-Laird, P.N., Kang, O.E. \& Leong, Y.C. (2012). On musical dissonance. Music Perception, 30(1), $19-35$.

Juhl, J., \& Routledge, C. (2013). Nostalgia bolsters perceptions of a meaningful self in a meaningful world. In J. Hicks \& C. Routledge (Eds.), The experience of meaning in life: Perspectives from the psychological sciences (pp. 213-226). New York: Springer Press.

Juslin, P. (2011). Music and emotion: Seven questions, seven answers. In I. Deliège \& J. Davidson (Eds.), Music and the mind: Essays in honour of John Sloboda (pp. 113-135). New York: Oxford University Press.

Juslin, P. (2013). What does music express? Basic emotions and beyond. Frontiers in Emotion Science, 4 (article 596), 1-14.

Juslin, P., Harmat, L., \& Eerola, T. (2013). What makes music emotionally significant? Exploring the underlying mechanisms. Psychology of Music, 42, 599-623.

Juslin, P. N., \& Laukka, P. (2003). Communication of emotions in vocal expression and music performance: Different channels, same code? Psychological Bulletin, 129, 770-814.

Juslin, P., \& Laukka, P. (2004). Expression, perception, and induction of musical emotions: A review and a questionnaire study of everyday listening. Journal of New Music Research, 33, 217-238.

Juslin, P., Liljeström, S., Västfjäll, D., Barradas, G., \& Silva, A. (2008). An experience sampling study of emotional reactions to music: Listener, music, and situation. Emotion, 8, 668-683. 
Juslin, P., \& Västfjäll, D. (2008). Emotional responses to music: The need to consider underlying mechanisms. Behavioral and Brain Sciences, 31, 559-621.

Kameoka, A., \& Kuriyagawa, M. (1969). Consonance theory. Part 1: Consonance of dyads. Journal of the Acoustical Society of America, 45, 1451-1459.

Kaminska, Z., \& Woolf, J. (2000). Melodic line and emotion: Cooke's theory revisited. Psychology of Music, 28, 133-153.

Kelly, B.L. (2001). "Ravel, (Joseph) Maurice." Grove Music Online. Edited by Deane Root. http://www.oxfordmusiconline.com (Accessed 17 November 2014).

Key, S. (1995). Sound and sentimentality: Nostalgia in the songs of Stephen Foster. American Music, 13(2), 145-166.

Krantz, G., Merker, B., \& Madison, G. (2004). Subjective reactions to musical intervals assessed by rating scales. In Proceedings of The Eighth International Conference on Music Perception and Cognition. Evanston, IL: Northwestern University.

Krumhansl, C. (1990). Cognitive foundations of musical pitch. New York: Oxford University Press.

Lahdelma, I., \& Eerola, T. (2014). Single chords convey distinct emotional qualities to both naïve and expert listeners. Psychology of Music, doi:10.1177/0305735614552006.

Laukka, P., Eerola, T., Thingujam, N. S., Yamasaki, T., \& Beller, G. (2013). Universal and culture-specific factors in the recognition and performance of musical affect expressions. Emotion, 13, 434-449.

Maher, T. (1980). A rigorous test of the proposition that musical intervals have different psychological effects. American Journal of Psychology, 93, 309-327.

Malin, Y. (2006). Metric displacement dissonance and romantic longing in the German Lied. Music Analysis, 25(3), 251-288.

Malmberg, C. F. (1918). The perception of consonance and dissonance. Psychological Monographs, 25(2), 93-133.

McDermott, J., Lehr, A., \& Oxenham, A. (2010). Individual differences reveal the basis of consonance. Current Biology, 20, 1035-1041.

Meyer, L.B. (1956). Emotion and meaning in music. Chicago: The University of Chicago Press.

Mitchell, D. (2002). Eternity or nothingness? Mahler's Fifth Symphony. In D. Mitchell \& A. Nicholson (Eds.), The Mahler Companion (pp. 236-325). New York: Oxford University Press.

Oelmann, H., \& Laeng, B. (2009). The emotional meaning of harmonic intervals. Cognitive Processing, 10(2), 113-131.

Pallesen, K., Brattico, E., Bailey, C., Korvenoja, A., Koivisto, J., Gjedde, A., \& Carlson, S. (2005). Emotion processing of major, minor, and dissonant chords: A functional magnetic resonance imaging study. Annals of the New York Academy of Sciences, 1060, 450-453.

Parncutt, R. (1993). Pitch properties of chords of octave-spaced tones. Contemporary Music Review, 9, 3550 .

Parncutt, R. (1989). Harmony: A psychoacoustical approach. Berlin, Germany: Springer-Verlag. 
Parncutt, R. (2011). The tonic as triad: Key profiles as pitch salience profiles of tonic triads. Music Perception, 28, 333-365.

Parncutt, R. (2012). Major-minor tonality, Schenkerian prolongation, and emotion: A commentary on Huron and Davis (2012). Empirical Music Review, 7, 118-137.

Parncutt, R. (2014). The emotional connotations of major versus minor tonality: One or more origins? Musicae Scientiae, 18(3), 324-353.

Parncutt, R., \& Hair, G. (2011). Consonance and dissonance in theory and psychology: Disentangling dissonant dichotomies. Journal of Interdisciplinary Music Studies, 5, 119-166.

Peretz, I. (2010). Towards a neurobiology of musical emotions. In Juslin, P. \& Sloboda, J. (Eds.), Handbook of music and emotion: Theory, research, applications (pp. 99-126). Oxford: Oxford University Press.

Schenker, H. (1906). Harmonielehre (Neue musikalische Theorien und Phantasien, Vol. 1). Stuttgart: J. G. Cotta.

Scherer, K. R. (1986). Vocal affect expression: A review and a model for future research. Psychological Bulletin, 99, 143-165.

Schoenberg, A. (1922/1990). Theory of harmony (Roy E. Carter, Trans.). London: Faber and Faber.

Schubert, E. (2010). Continuous self-report methods. In Juslin, P. \& Sloboda, J. (Eds.), Handbook of music and emotion: Theory, research, applications (pp. 223-253). Oxford: Oxford University Press.

Sedikides, C., Wildschut, T., Arndt, J., \& Routledge, C. (2008). Nostalgia: Past, present, and future. Current Directions in Psychological Science, 17, 304-307.

Sedikides, C., Wildschut, T., \& Baden, D. (2004). Nostalgia: Conceptual issues and existential functions. In J. Greenberg, S. Koole, \& T. Pyszczynski (Eds.), Handbook of experimental existential psychology (pp. 200-214). New York, NY: Guilford Press.

Spencer, H. (1857). The origin and function of music. Fraser's Magazine, 56, 396-408.

Tagg, P. (1993). 'Universal' music and the case of death.

http://www.tagg.org/articles/xpdfs/deathmus.pdf (Accessed 17 November 2014).

Tagg, P., \& Clarida, B. (2003). Ten little title tunes: Towards a musicology of the mass media. New York: The Mass Media Music Scholars' Press.

Taruffi, L., Koelsch, S. (2014). The paradox of music-evoked sadness: An online survey. PLoS ONE 9(10): e110490. doi: 10.1371/journal.pone.0110490

Terhardt, E. (1974). On the perception of periodic sound fluctuations (roughness). Acustica, 30, 201-213.

The New Oxford Dictionary of English (1998). Oxford: Oxford University Press.

Tillman, B., Bharucha, J.J., \& Bigand, E. (2000). Implicit learning of tonality: A self-organizing approach. Psychological Review, 107(4), 885-913.

Toch, E. (1948). The shaping forces in music. New York: Criterion Music Corp. 
Tramo, M.J., Cariani, P.A., Delgutte, B., \& Braida, L.D. (2001). Neurobiological foundations for the theory of harmony in western tonal music. Annals of the New York Academy of Sciences, 930, 92-116.

Vassilakis P. N. (2001). Perceptual and physical properties of amplitude fluctuation and their musical significance (Unpublished doctoral dissertation). University of California, Los Angeles.

Werman, D. S. (1977). Normal and pathological nostalgia. Journal of the American Psychoanalytic Association, 25, 387-398.

Virtala, P., Huotilainen, M., Partanen, E., Fellman, V., \& Tervaniemi, M. (2013). Newborn infants' auditory system is sensitive to Western music chord categories. Frontiers in Auditory Cognitive Neuroscience, 4, 462, 1-10.

Wildschut, T., Sedikides, C., Arndt, J., \& Routledge, C. (2006). Nostalgia: Content, triggers, functions. Journal of Personality and Social Psychology, 91, 975-993.

Wildschut, T., Sedikides, C. \& Routledge, C. (2008). Nostalgia: From cowbells to the meaning of life. The Psychologist, 21, 20-23.

Zentner, M., Grandjean, D., \& Scherer, K. (2008). Emotions evoked by the sound of music: Characterization, classification, and measurement. Emotion, 8, 494-521.

Zentner, M., \& Eerola, T. (2010). Self-report measures and models of musical emotions. In Juslin, P. \& Sloboda, J. (Eds.), Handbook of music and emotion: Theory, research, applications (pp. 187-221). Oxford: Oxford University Press.

Zentner, M. \& Kagan, J. (1996). Perception of music by infants. Nature, 383, 29. 